\title{
An improved method for quantitative ChIP studies of nuclear receptor function
}

\author{
Ann Louise Hunter1, Natasha Narang1, Matthew Baxter1,2, David W Ray,1,2,3 and Toryn M Poolman ${ }^{1,2}$ \\ ${ }^{1}$ Faculty of Biology, Medicine and Health, University of Manchester, Manchester, UK \\ 2Oxford Centre for Diabetes, Endocrinology and Metabolism, University of Oxford, Oxford, UK \\ ${ }_{3}^{3}$ IHRR Oxford Biomedical Research Centre, John Radcliffe Hospital, Oxford, UK
}

Correspondence should be addressed to A L Hunter: louise.hunter@manchester.ac.uk

\begin{abstract}
Chromatin immunoprecipitation (ChIP) is a valuable tool for the endocrine researcher, providing a means to measure the recruitment of hormone-activated nuclear receptors, for example. However, the technique can be challenging to perform and has multiple experimental steps, risking introduction of error at each. The data produced can be challenging to interpret; several different methods are commonly used for normalising data and thus comparing between conditions. Absolute, sensitive quantification of protein-bound DNA is important for correct interpretation of the data. In addition, such
\end{abstract} Key Words

- ChIP

- digital PCR

- ddPCR

- troubleshooting

- nuclear receptors

\section{Introduction}

Analysis of protein-DNA interactions, using chromatin immunoprecipitation (ChIP), has provided much new information in the understanding of signalling pathways key to cancer, metabolism and development. For example, the interaction of the oestrogen receptor (ER) with the chromatin landscape can be used to predict treatment outcomes and patient survival in breast cancer (Ross-Innes et al. 2012). The locations and regulation of enhancer sites important to normal human pancreas development have been characterised using ChIP approaches (Cebola et al. 2015).

ChIP is a widely-used but challenging technique (Schubert 2018). We illustrate a typical workflow in Fig. 1. Fixed chromatin prepared from cells or tissues is fragmented (with sonication or enzymatic digestion) to 200-1000bp lengths. Then, using an antibody against the transcriptional regulator or histone modification of interest, protein-bound DNA is immunoprecipitated. After de-crosslinking, the enriched DNA is purified and can be analysed by quantitative polymerase chain reactions (ChIP-qPCR) or by next-generation sequencing (ChIP-seq).

There are inherent problems with ChIP quantification, and these are especially marked when studying nuclear receptors, which may be highly dynamic, low-abundance proteins; we have encountered many such problems when studying the action of the glucocorticoid receptor (GR).

The final amount of DNA from ChIP reactions may be extremely small. In ChIP-qPCR, a positive sample (i.e. ChIP DNA containing the bound region of interest) may still have a CT (threshold cycle) value well above 30, at which levels background may obscure signal. The low yield of ChIP reactions means that a considerable number of cells, or amount of tissue, may be required to study some targets. It is possible to carry out ChIP successfully on very small samples, such as needle 


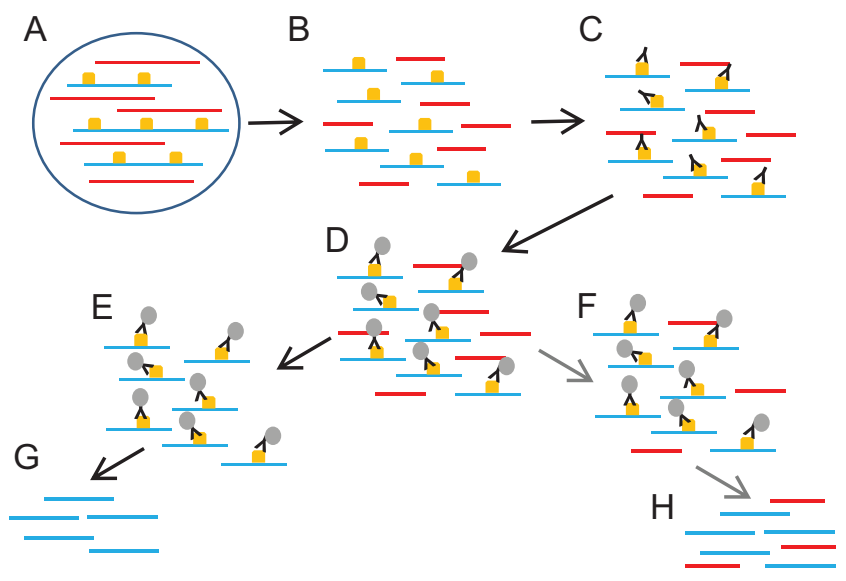

Figure 1

Diagram illustrating the ChIP reaction and potential sources of background. Target-bound DNA is shown in red, background (nonspecifically-bound) DNA in blue. (A) Following fixation and cell lysis, the chromatin sample is sonicated to (B) 200-1000 bp size. (C) Using an antibody, the target of interest (e.g. transcription factor or histone mark) is immunoprecipitated. (D) Immune complexes are bound using protein A/G (either magnetic or agarose based). The sample is then extensively washed, to remove as much non-specific DNA as possible; however, the level of background DNA may differ between samples ( $E$ and F). Moreover, the elution step can also influence the levels of background DNA, depending on the buffer conditions used, which results in a variable level of DNA in each sample ( $G$ and $H)$.

biopsies from breast cancer patients (Zwart et al. 2013), but such studies tend to be of highly robust targets (e.g. ER over-expressed in cancer) and can be far more challenging in the physiological state. Low yield is a particular problem for ChIP-sequencing experiments, as library preparation kits for whole genome sequencing are typically optimised for quantities of DNA in the nanogram range. We find that, in order to recover sufficient (e.g. $5 \mathrm{ng}$ ) ChIP DNA for ChIP-sequencing of histone marks, at least $10-30 \mu \mathrm{g}$ of chromatin (as measured by the concentration of the sonicated input sample) is needed per immunoprecipitation (IP) reaction. For transcription factor (e.g. GR) ChIP-seq, multiple (e.g. three) IP reactions are needed, totalling 75-90 $\mu \mathrm{g}$ of chromatin. If different experimental groups are to be compared, then accurate quantification of input chromatin is essential to ensure that identical amounts are added to each IP reaction.

A further challenge is posed by the analysis of purified ChIP DNA to determine the enrichment of a bound region of interest. This can be performed in several ways and each has their pitfalls.

One method is to carry out an additional IP reaction with non-specific IgG in place of the experimental antibody. Using qPCR, the abundance of the region of interest is quantified from both IPs, and fold enrichment expressed as experimental antibody over non-specific IgG. However, the DNA from the IgG IP is often too low to measure reliably, and thus can give false enrichment.

Another means is to quantify a known, bound (positive) region in the input sample, and in the ChIP DNA, and calculate the ChIP enrichment as a percentage of the input signal. This approach does have some correcting potential, but does not distinguish if the target region is being specifically or non-specifically bound by the antibody. This is because it ignores the background signal, that is, the quantity of DNA from regions of the genome where no binding should be observed (negative regions).

Determining the signal in the ChIP DNA from a negative region and using that to determine the fold enrichment of the positive region is a more appropriate measure, which does correct for non-specific binding. However, in an IP with a high signal-to-noise ratio, negative regions may be of very low abundance, and thus quantifying them with qPCR may not be possible. Moreover, standard curves need to be run with the primers for each target.

Combined, these problems raise concern that ChIP experiments may have high technical variability, regardless of biological variation, and that the success of a ChIP experiment, before moving to costly ChIP sequencing, may be difficult to determine. There has been an unmet need for reliable techniques, more robust than qPCR and less costly than ChIP-seq. ChIP experiments designed for qPCR and those for ChIP-seq are on very different scales, there being a gap for technologies that will provide more information on protein-DNA interactions across time series data, affected by inhibitors, or affected by SNPs, for example.

We suggest that this space could be occupied by combining ChIP with droplet digital PCR (ddPCR) (Hindson et al. 2011). Following purification, ChIP DNA is added to ddPCR mastermix with primers directed at positive or negative regions. The reaction mixture is then dispersed into droplets (typically 20,000 per $20 \mu \mathrm{L}$ of reaction mix) suspended in oil, using specialised equipment (a Droplet Generator). The stably partitioned sample then undergoes thermal cycling in a 96-well plate, akin to any other PCR reaction. The fluorescence signal from each individual droplet is subsequently read by an automated Droplet Reader, as the droplets are aspirated from the wells. Droplets that contain amplified template are counted as 'positive', those that do not are deemed 'negative'. Thus, the amount of template can be quantified absolutely, rather than relative quantification using a standard curve or normalising to a reference target.

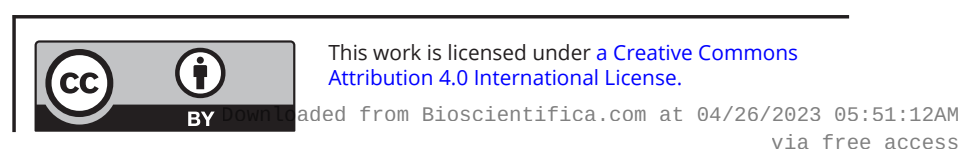


The performance of ddPCR and qPCR for gene expression analysis has been directly compared (Taylor et al. 2017), with the authors reporting comparable performance in the quantification of samples with minimal or no contamination. However, in samples with variable levels of contamination, ddPCR results remained consistent, whilst qPCR produced inconsistent and markedly variable results. The authors conclude that ddPCR is the preferable strategy in the quantification of low abundance targets where dilution to minimise contaminants is not possible (Taylor et al. 2017). As discussed earlier, ChIP unavoidably requires the quantification of low abundance targets.

Exploiting these advantages of digital PCR, the technique has been combined with ChIP-related techniques such as chromosome conformation capture (3C) (Link et al. 2013, Du \& Wang 2016), and in quantifying binding of Cas9 endonuclease to its targets (Chen et al. 2017). In recent years, ChIP-ddPCR itself has been employed to study histone marks (Kim et al. 2016) and the binding of transactivator protein CIITA (Wong et al. 2014). These papers have expressed ChIP signal as a proportion of input signal.

Our group has also used ChIP-ddPCR to examine the effect of clock protein REVERB $\alpha$ on GR binding (Caratti et al. 2018). Included in this, however, is the combination of ChIP-ddPCR with the use of an internal spike-in control, rather than normalising to input signal or IgG control. This has been part of our efforts to improve the reproducibility of our ChIP experiments. In the ChIP spike-in strategy, cells or chromatin from a foreign species (e.g. Drosophila melanogaster) are added to the IP reaction, alongside the experimental chromatin (Bonhoure et al. 2014, Orlando et al. 2014, Egan et al. 2016). The most recent evolution of this technique (which we employ) also adds an antibody specific to a target on the foreign chromatin (Egan et al. 2016), so that two separate IP reactions are essentially occurring simultaneously within the one tube. Purified ChIP DNA therefore contains material from both the experimental species and the spike-in control; the signal from each of these can be quantified by ddPCR, and technical variation reduced by normalising experimental signals to the spike-in signal.

Here, we share our experience with the technique of ChIP-ddPCR plus spike-in normalisation, which we believe demonstrates its value in accurately quantifying ChIP DNA, in assessing the quality of a ChIP experiment, in troubleshooting the ChIP workflow, and in reducing variability, thus producing more robust data.

\section{Materials and methods}

\section{Animals}

All in vivo experiments were conducted in accordance with local requirements and with the UK Animals (Scientific Procedures) Act 1986. All procedures were approved by the University of Manchester Animal Welfare and Ethical Review Body (AWERB). C57BL/6 mice were purchased from Envigo (Hillcrest, UK) and group-housed with ad libitum access to food and water. For dexamethasone treatment, mice were injected with $1 \mathrm{mg} / \mathrm{kg}$ dexamethasone (cat. D2915 - Sigma-Aldrich) by the intraperitoneal route, $1 \mathrm{~h}$ prior to being killed.

\section{Sample preparation}

ChIP assays were carried out on flash-frozen mouse kidney or liver tissue using the ChIP-IT High-Sensitivity Kit (cat.53040 - Active Motif, Carlsbad, USA). The assay was carried out as per the manufacturer's recommendations with slight modifications as described. Following formaldehyde-assisted chromatin fixation, tissue was homogenised using the handheld TissueRuptor (Qiagen). For kidney tissue, cells were then passed through a cell strainer in between PBS washes to obtain more uniform cell suspensions, due to the fibrosity of kidney tissue. Nuclei release was achieved by dounce homogenisation using a tight pestle. Chromatin was sheared by sonication using the EpiShear Probe Sonicator (Active Motif) with a $3.5 \mathrm{~mm}$ probe. Sonicating conditions were as follows: amplitude 37\%, pulse for 30 s on and 30 s off, for a total sonication time of $2 \mathrm{~min}$; this cycle was repeated eight times per sample. To prevent overheating and denaturation, samples were kept on ice between cycles, and the EpiShear-cooled sonication platform was used and replaced regularly throughout the sonication process.

Following sonication, input DNA was generated by treating samples with RNase $\mathrm{A}$ and proteinase $\mathrm{K}$, heating, and then precipitating with ethanol. Input DNA concentrations were determined by both spectrophotometric (Nanodrop 2000 - Thermo Fisher Scientific) and fluorometric (Qubit HS or BR dsDNA assayThermo Fisher Scientific) quantification. A diluted aliquot of each input was electrophoresed by TapeStation (Agilent Technologies) to assess chromatin shearing efficacy.

Immunoprecipitation reactions were performed on prepared chromatin by incubation with antibodies for histone H3K27ac (cat.39133 - Active Motif), histone H3K27me3 (cat. 39155 - Active Motif) and GR (cat.240501AP - Proteintech Group, Rosemont, USA;

This work is licensed under a Creative Commons Attribution 4.0 International License. 
cat.3660 (D8H2) - Cell Signaling Technology), on an end-to-end rotator overnight at $4^{\circ} \mathrm{C}$. Incubation with MagReSyn protein G beads (cat.MR-PRG002 - ReSyn Biosciences, Gauteng, South Africa) on an end-to-end rotator was then performed for $3 \mathrm{~h}$ at $4^{\circ} \mathrm{C}$. Beads (with antibody-chromatin complexes bound) were then washed, and ChIP material was eluted from the beads using Active Motif proprietary kit buffer (unless stated otherwise). ChIP DNA was treated with proteinase $\mathrm{K}$ and heated for $2.5 \mathrm{~h}$ to reverse cross-links, and then purified on a column using $36 \mu \mathrm{L}$ proprietary Active Motif DNA purification elution buffer. For spike-in normalisation, spike-in (Drosophila melanogaster) chromatin (cat.53083 - Active Motif) and its specific antibody (cat.61686 - Active Motif) were added simultaneously to each IP reaction.

\section{ChIP-qPCR}

Two microliters of ChIP DNA were used for each qPCR reaction. Standard curves of input DNA, ranging from 0.005-50 ng of DNA, were generated for each primer set used, ensuring that the CT values generated are measuring a real quantity of DNA. Assays were carried out on a fast (40min) cycle using GoTaq qPCR, on an Applied Biosystems StepOne system (Thermo Fisher Scientific).

\section{ChIP-ddPCR}

Each ddPCR assay mix contained $2 \mu \mathrm{L}$ ChIP DNA, $10 \mu \mathrm{L}$ QX200 ddPCR EvaGreen Supermix (Bio-Rad Laboratories) and $0.8 \mu \mathrm{L}$ primer mix, with water added to reach a final reaction volume of $22 \mu \mathrm{L}$. Twenty microliters of each assay mix was loaded into the sample well of a droplet generator cartridge (Bio-Rad) and $70 \mu \mathrm{L}$ of droplet generation oil (Bio-Rad) loaded into each of the oil wells. The cartridge was then transferred to the QX200 Droplet Generator (Bio-Rad). Once droplet generation was complete, the cartridge was removed from the Droplet Generator and $40 \mu \mathrm{L}$ of the droplet emulsion was transferred to a standard 96-well PCR plate. Following droplet loading, the 96-well plate was transferred to the PX1TM Plate Sealer (Bio-Rad) to heat seal the plate with pierceable foil (Bio-Rad). The plate was then transferred to a conventional thermal cycler (Bio-Rad), with cycling conditions as follows: $95^{\circ} \mathrm{C}$ for $5 \mathrm{~min}$, and then $95^{\circ} \mathrm{C}$ for $30 \mathrm{~s}$ and $60^{\circ} \mathrm{C}$ for $1 \mathrm{~min}$ for 40 cycles, followed by $4^{\circ} \mathrm{C}$ for $5 \mathrm{~min}$ and finally $90^{\circ} \mathrm{C}$ for $5 \mathrm{~min}$. Droplets were then aspirated and read by the QX200 Droplet Reader (Bio-Rad).

\section{ChIP primers}

The Mouse-Negative Control Primer Set 2 (cat.71012) and Mouse-Positive Control Primer Set (Gapdh promoter) (cat.71016) are proprietary products obtained from Active Motif. Tyrosine aminotransferase (Tat) ChIP primers were published by Phuc Le et al. (2005): forward primer 5'-CGCAAACAACAGGAAGCCTAA-3', reverse primer 5'-CATGACACCCAAAAGCCTCTC-3'. The ChIP primer for chr17:85052667-85052752 (Slc3a1 intron) is of original design and was manufactured by Eurofins Genomics (Ebersberg, Germany): forward primer 5'-TGAGTGCTTATCTACAGGGTTCG-3', reverse primer 5'-AGATTCTTCCССТTCTACACACA-3'.

\section{Data analysis}

ddPCR data were analysed using the QuantaSoft software package (version 1.7 - Bio-Rad). Droplet data are either shown raw (copies $/ \mu \mathrm{L}$ ), $\log _{2}$-transformed or represented as the fold enrichment over the negative control primer.

\section{Results}

\section{The ChIP process and potential for error}

The ChIP process is complex, and presents difficulties, especially with low abundance targets or limited input material. Figure 1 shows a typical ChIP reaction; after cross-linking and DNA fragmentation, the target of interest is enriched using an antibody and then captured with protein A or G (either magnetic or agarose based) or newer technologies such as ChromaTrap(R) (Fig. 1A, B, C and D). After extensive washing, a mixture of specific and nonspecific DNA remains, each component of the enrichment (antibody and capture method) is likely to contribute to the non-specific pool. The background DNA in each sample may not uniform (Fig. 1E and F), and thus, its contribution to the eluted DNA (Fig. $1 \mathrm{G}$ and $\mathrm{H}$ ) potentially masks the enrichment of a truly bound region. This is particularly important when samples are prepared for ChIP-seq.

\section{Combining ddPCR with the ChIP workflow}

To test the ddPCR reactions with ChIP DNA, we started with chromatin prepared from mouse tissue and performed immunoprecipitation reactions with normal IgG or H3K27ac and H3K27me3 antibodies. ChIP targeting histone marks typically yields greater quantities of output DNA than transcription factor ChIP. The Gapdh promoter was used as a positive control region and was tested against

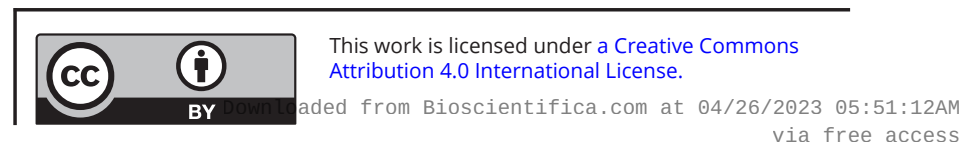


a negative control region (a gene desert on chromosome 17). Input DNA samples showed similar abundance of the positive and negative regions (Fig. 2A(i)), demonstrating equivalent efficiency of the two primer sets. The signal from both regions was low but detectable in the IgG IPs (Fig. 2A(ii)). The H3K27ac signal was high at the Gapdh promoter (Fig. 2A(iii)) and H3K27me3 signal (a repressive mark) was low (Fig. 2A(iv)), indicating a gene permissive to transcription. The total amounts of DNA recovered from these IPs (measured using a Qubit HS dsDNA assay) were 6, 25 and $75 \mathrm{ng}$ in total for IgG, H3K27ac and H3K27me3 respectively.

Moving to performing IPs with an antibody against a transcription factor (GR), we quantified the DNA from a well-characterised GR-binding site near the Tat promoter. This signal was clearly enriched over the Tat signal from an IgG IP (Fig. 2B(i) and (ii)) and over the signal from a negative region quantified from the GR IP (Fig. 2B(ii)). We were also able to determine the effect of treatment with dexamethasone (a synthetic GR ligand) on GR binding at Tat (Fig. 2B(iii)).

We made a comparison between ddPCR copies $/ \mu \mathrm{L}$ and qPCR CT values for our positive control site (Tat promoter), and for the negative region control region, across IgG, anti-GR and anti-H3K27ac IPs with liver chromatin. ddPCR values correlated well with qPCR results for both the positive (Fig. 3A) and negative regions (Fig. 3B). However, the resulting fold change values varied more widely (Fig. 3C), with poorer correlation, and fold changes derived from qPCR data being higher than those from ddPCR data (Supplementary Table 1 provides the original data and calculated fold changes, see section on supplementary data given at the end of this article). Note that many of the CT values - especially for the negative region - lie above 32, a level at which direct comparison has found qPCR to demonstrate greater variability than ddPCR (Taylor et al. 2017). If used as the denominator for a fold change calculation, the negative signal must be quantified with accuracy or else the fold change may be falsely inflated or deflated. Here, ddPCR offers an advantage.

\section{Troubleshooting ChIP experiments with ddPCR}

Because ChIP-ddPCR provides absolute rather than relative quantification of ChIP DNA, we have found it to be a useful means of troubleshooting difficult ChIP experiments. There is no need to quantify two signals,
A

(i) Input sample

(ii) IgG ChIP

(iii) H3K27ac ChIP

(iv) H3K27me3 ChIP
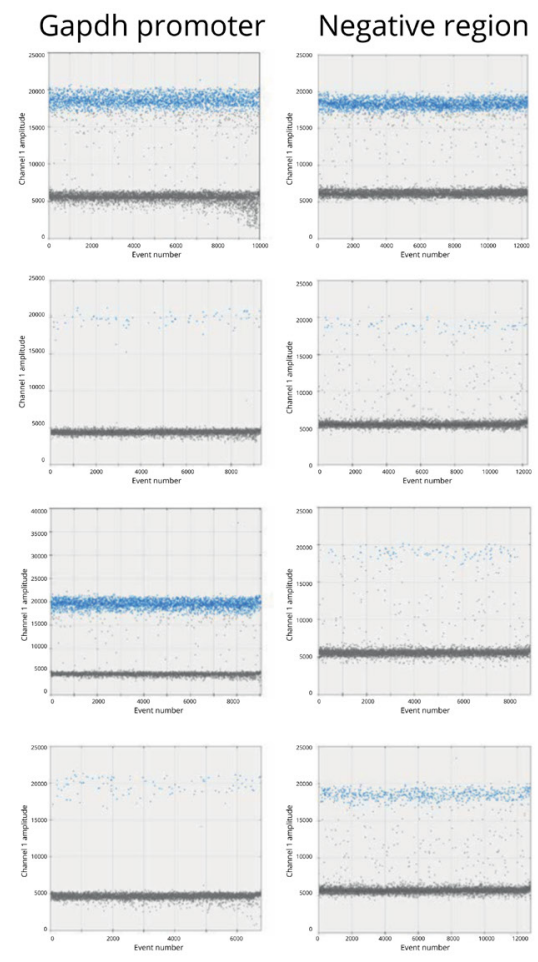

B

(i) IgG ChIP

(ii) GR ChIP
vehicle-treated
sample
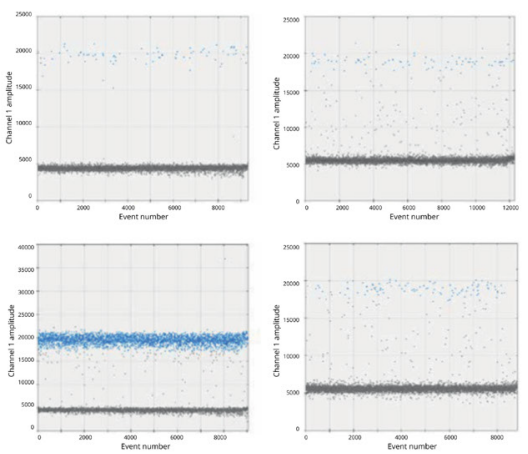

(iii) GR ChIP dexamethasonetreated sample
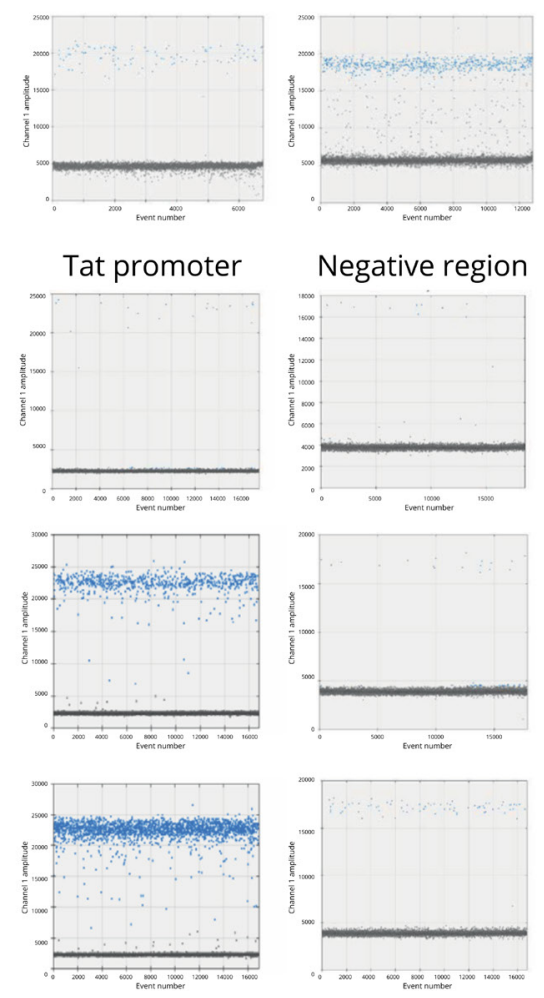

Figure 2

Typical output of ChIP droplet digital (dd)-PCR assays. (A) $30 \mu \mathrm{g}$ of mouse kidney chromatin was sonicated and ChIP assays were performed on the Gapdh promoter or a negative control region. Input DNA sample shown in (i); immunoprecipitations were carried out with (ii) IgG, (iii) anti-H3K27ac antibody and (iv) anti-H3K27me3 antibody. (B) $30 \mu \mathrm{g}$ of sonicated mouse kidney chromatin was assayed for glucocorticoid receptor (GR) binding to the tyrosine aminotransferase (Tat) promoter or a negative binding region. IgG (i) or GR was immunoprecipitated from mice dosed with saline (ii) or $1 \mathrm{mg} / \mathrm{kg}$ dexamethasone (iii). ddPCR 1D plots are shown; positive reaction droplets are in blue, and negative ones in grey.
(C) 2019 The authors Published by Bioscientifica Ltd. Printed in Great Britain

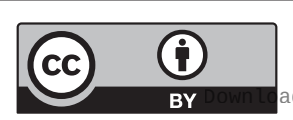

This work is licensed under a Creative Commons Attribution 4.0 International License.

ded from Bioscientifica.com at $04 / 26 / 2023$ 05:51:12AM 

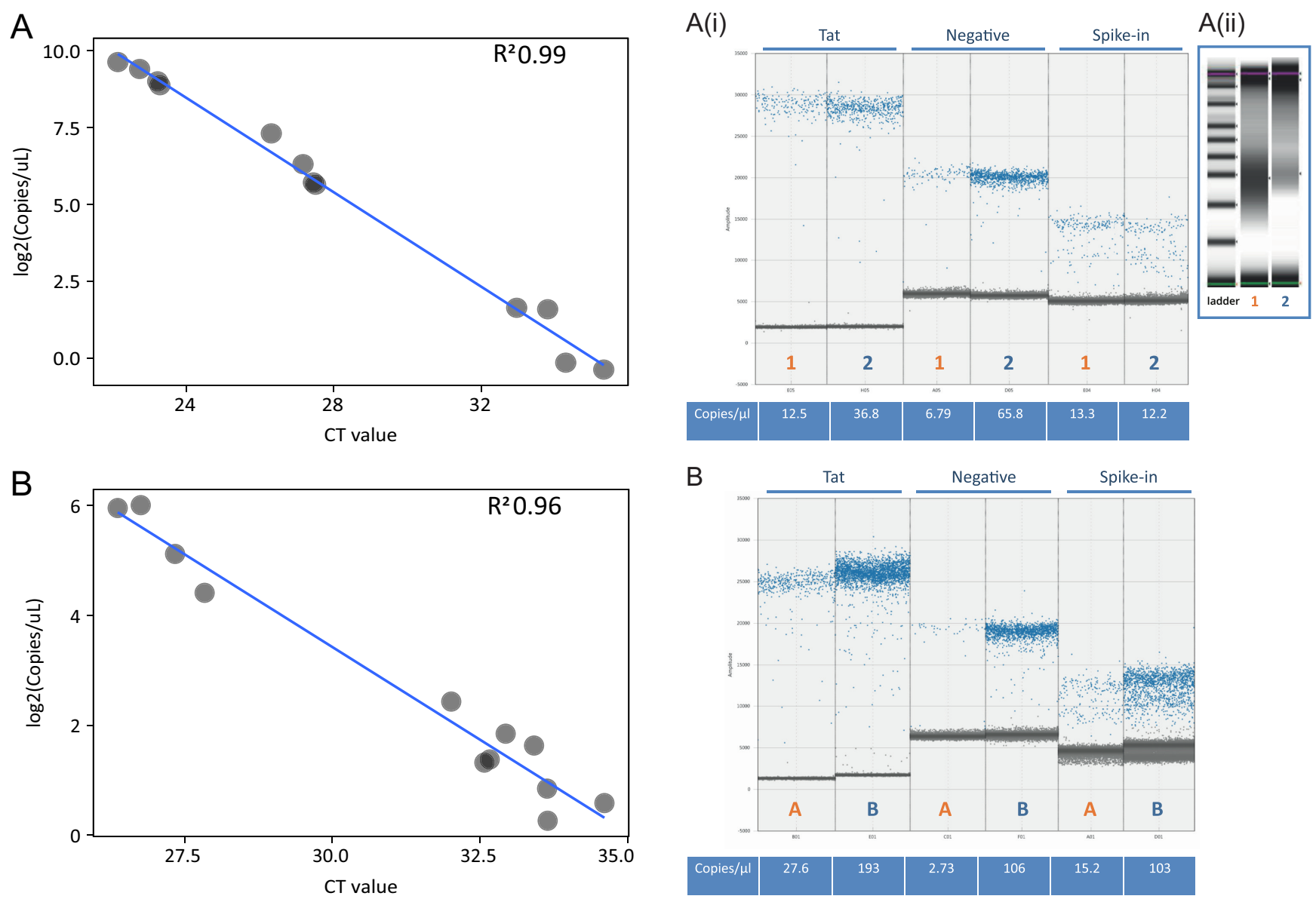

B
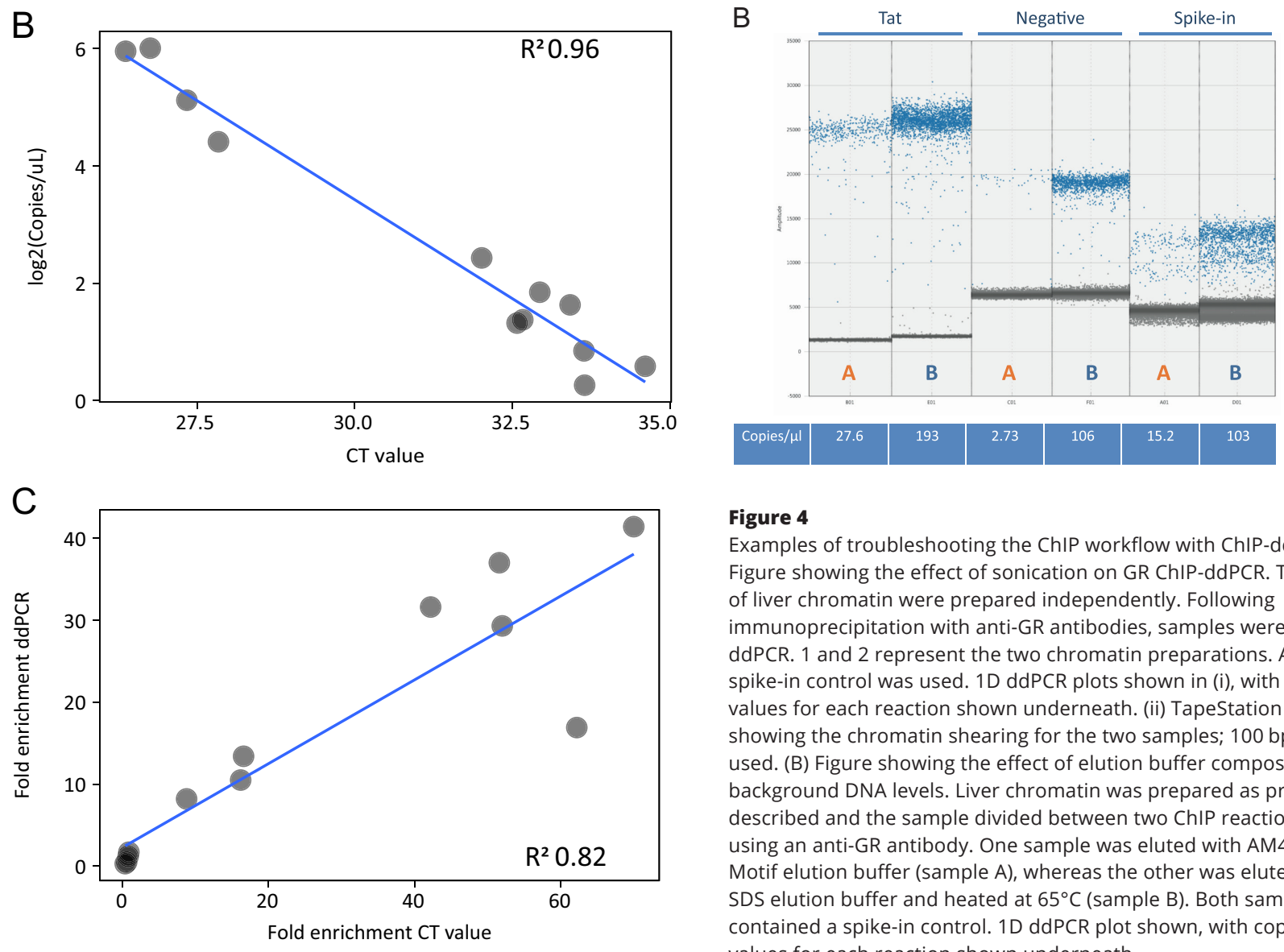

Figure 3

Graphical comparison of ChIP-ddPCR and ChIP-qPCR. (A) qPCR and ddPCR comparisons for the Tat promoter and (B) negative control region. (C) Fold enrichment was determined for both methods as fold change over negative region. H3K27ac, GR and IgG ChIP samples are plotted. Graphs show copies/ $\mu \mathrm{L}$ plotted against CT value. $R^{2}$ values were determined by linear regression.

or rely on a standard curve, and thus, the source of error is easier to identify. We demonstrate this here with two exemplar experiments.

\section{Figure 4}

Examples of troubleshooting the ChIP workflow with ChIP-ddPCR. (A) Figure showing the effect of sonication on GR ChIP-ddPCR. Two samples of liver chromatin were prepared independently. Following

immunoprecipitation with anti-GR antibodies, samples were analysed by ddPCR. 1 and 2 represent the two chromatin preparations. A Drosophila spike-in control was used. 1D ddPCR plots shown in (i), with copies $/ \mu \mathrm{L}$ values for each reaction shown underneath. (ii) TapeStation image showing the chromatin shearing for the two samples; 100 bp ladder used. (B) Figure showing the effect of elution buffer composition on background DNA levels. Liver chromatin was prepared as previously described and the sample divided between two ChIP reactions each using an anti-GR antibody. One sample was eluted with AM4 Active Motif elution buffer (sample A), whereas the other was eluted with a $1 \%$ SDS elution buffer and heated at $65^{\circ} \mathrm{C}$ (sample B). Both samples contained a spike-in control. 1D ddPCR plot shown, with copies/ $\mu \mathrm{L}$ values for each reaction shown underneath.

Figure 4A (i) illustrates ddPCR quantification following IPs, 1 and 2. Quantity of input chromatin and choice of antibody were identical for both reactions. The signal from the positive region (Tat promoter) is clearly higher in two. In the absence of other information, this could suggest more specific binding of the positive region in 2, perhaps because of biological variation. However, quantification of a negative region reveals that the background signal is nearly tenfold greater in 2 , and

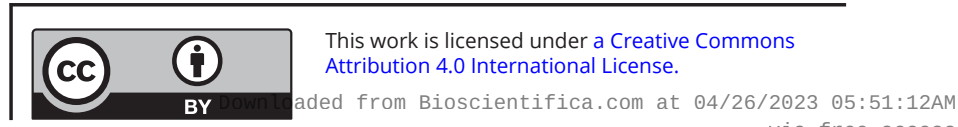


hence, fold enrichment of negative over positive signal is actually poorer. Background signal, reflecting DNA not bound by the protein of interest, is influenced by quality of chromatin preparation, the efficiency of the IP reaction, the washing of the beads and the elution of the ChIP DNA from the beads in the final stages. High background signal often leads to poorer fold enrichment, and thus, falsenegative results.

Further useful information can be gained from the quantification of the spike-in control, which is independent of the chromatin preparation, but permits normalisation between samples for IP efficiency, washing stringency, DNA elution and purification and, in the case of ChIP-seq, library amplification by PCR (Chen et al. 2015, Egan et al. 2016). For both 1 and 2, spike-in DNA quantities are very similar (Fig. 4A (i)), suggesting that the high background signal results from an earlier step in the process. Assessing the quality of the input chromatin indeed reveals that the shearing is poorer for sample 2 (Fig. 4A (ii)), the likely source of the error.

By comparison, Fig. 4B illustrates two further ChIP reactions, $\mathrm{A}$ and $\mathrm{B}$. Chromatin preparation and IP set up (including spike-in) was identical for each. The positive signal is far higher in B, but so is the negative signal. This time, quantification of the spike-in is different; the spike-in signal in B is also much higher. Therefore, the high background signal and poorer fold enrichment seen in B likely result from a difference introduced at the IP stage or later. In fact, elution conditions were changed between $\mathrm{A}$ and $\mathrm{B}$, with a different (harsher) elution buffer (1\% SDS) being used for sample B.

\section{Adding an internal spike-in control to ChIP-ddPCR}

Including absolute quantification of a spike-in signal in our ChIP-ddPCR protocol has also provided an illustration of the inherent inter-experiment variation, which affects ChIP experiments. Using ChIP-ddPCR, we quantified the spike-in signal across 56 ChIP reactions, conducted on five separate occasions (Fig. 5A). Of note, the quantity of spike-in chromatin and antibody were unchanged across all the experiments, as were the quantities of experimental chromatin (mouse liver) and experimental antibody (anti-GR), as was the operator. Despite this, the marked variation in spike-in counts (up to 30-fold) highlights the potential for other technical factors to influence ChIP results. In this situation, as chromatin preparations were of similar quality, variation in IP efficiency, washing stringency and DNA elution (despite strict adherence to a protocol) are the likely culprits. Printed in Great Britain
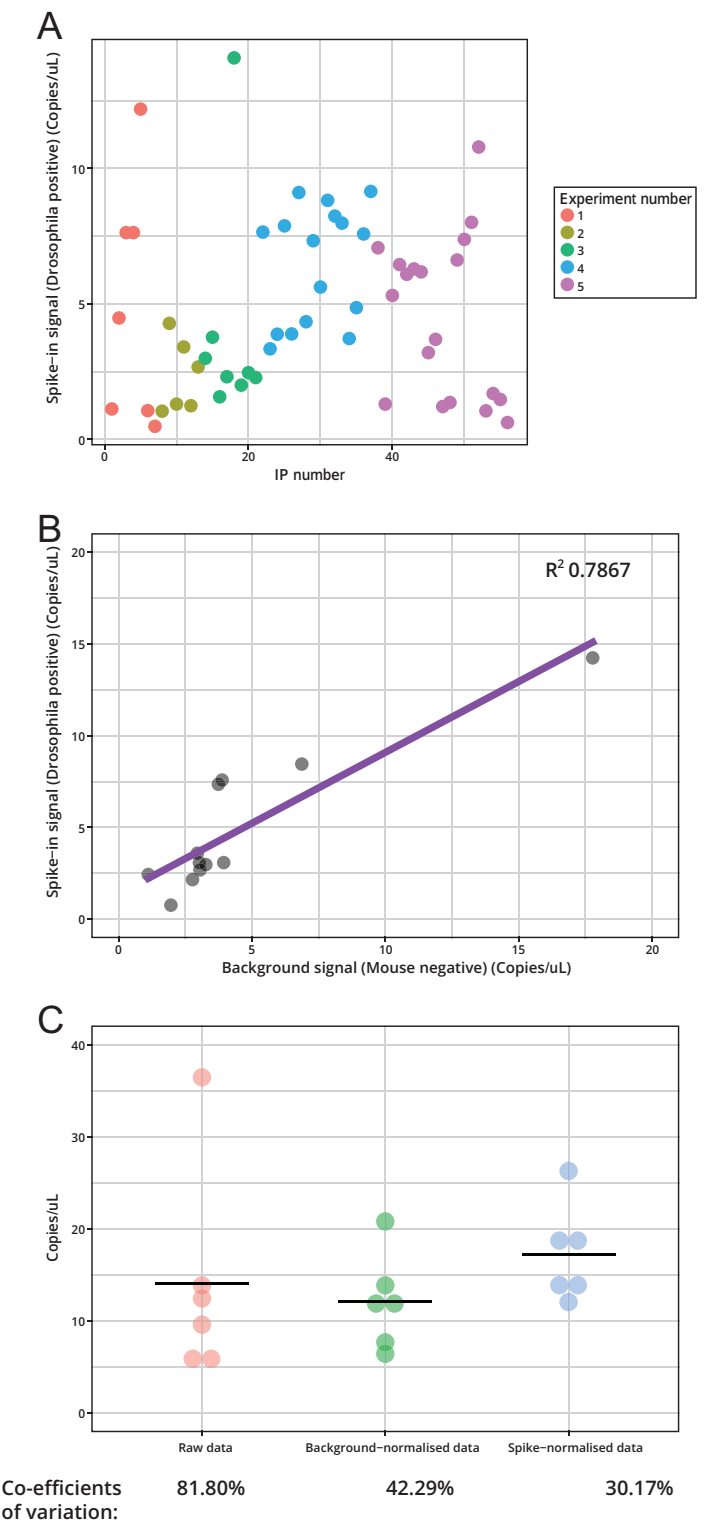

Figure 5

Combining spike-in with ddPCR to improve ChIP experiments. (A) Spike-in reveals inherent variation in ChIP experiments. Graph plots spike-in values (copies $/ \mu \mathrm{L}$ ) for 56 ChIP reactions (identified by IP number). The 56 ChIP reactions were conducted across 5 separate experiments (each shown in a separate colour) all using the same technical conditions (antibodies, elution buffer, quantities of experimental and spike-in DNA). (B) Spike-in values correlate positively with the background signal from ChIP reactions. Graph plots spike-in signal (quantified with Drosophila positive primer set) against background signal (Mouse negative primer set) across simultaneous ChIP assays performed in 12 samples of mouse liver chromatin. $R^{2}$ value determined by linear regression. (C) Normalising data to an internal spike-in control reduces relative variability within an experimental group. Graph plots ChIP-ddPCR counts for GR ChIP signal at a known GR-binding site on chromosome 17 (S/c3a1 intron), in six samples of dexamethasonetreated mouse liver chromatin. Individual data points plotted, line at mean. Spike-in signal and background signal were also assayed in each sample. Raw data were then normalised to either background or spike-in signal, using the method illustrated in Supplementary Fig. 1. Coefficients of variation are shown below each group of data.

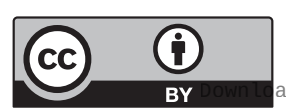

This work is licensed under a Creative Commons Attribution 4.0 International License. 
We were also interested to compare signal from an internal spike-in control with that from a background region. In theory, each should correlate with the other to an extent, both are influenced by the efficiency of the IP reaction and of the washing and elution steps. However, background signal is influenced by the quality of the experimental chromatin preparation, whilst spike-in is not. In turn, the utility of the spike-in is dependent on careful measurements of chromatin quantity when setting up IP reactions. We quantified both spike-in signal and background signal in 12 simultaneously prepared and simultaneously performed ChIP reactions (separate from the 56 discussed earlier) containing identical masses of spike-in chromatin and mouse liver chromatin (quantified by fluorometric means) (Fig. 5B). Correlation was good ( $R^{2}$ value, as determined by linear regression, of 0.7867 ) but not perfect. It is also of note that spike-in counts showed wide variation in this experiment too, with a 19-fold difference between smallest and largest spike-in signals. There was a similarly large (16-fold) difference across background signals, despite IPs being performed simultaneously by the same operator.

We then tested whether normalisation to either background or spike-in signal produced different results. In six samples of chromatin from mouse liver treated with dexamethasone (six biological replicates), we assayed GR ChIP signal at a known GR-binding site on chromosome 17 (intronic region of Slc3a1 gene), alongside background signal and spike-in signal. We then used either background or spike-in signals to produce normalisation factors (raw and normalised data shown in Supplementary Table 2, calculation workflow illustrated in Supplementary Fig. 1, based on recommendations in spike-in reagent (Active Motif) product literature) and observed that spike-in normalisation had a greater effect in reducing relative variability within an experimental group (Fig. 5C). The internal spike-in control does add a small amount of time and cost to the existing ChIP-ddPCR workflow, but has much to offer in reducing the technical variation.

\section{Discussion}

ChIP is demanding, taking up to a week in the laboratory, with many potential sources of error. Nonetheless, it is unrivalled for the study of protein-DNA interactions (Jordán-Pla \& Visa 2018). ddPCR, a recent advancement in DNA detection, can detect nucleic acids at absolute levels. We have added ddPCR to our ChIP workflow for this capability. Since ChIP-PCR relies on a comparison between signals, whether that be from an IgG IP, a negative region, a spike-in or a combination of these, being able to absolutely measure signal in those samples is important for determining the overall fold change and its significance. Here, we believe that ChIP-ddPCR offers greater reliability than ChIP-qPCR, as it does for quantifying gene expression changes (Taylor et al. 2017).

By applying ddPCR to ChIP, in combination with an internal spike-in control strategy, we have also been able to troubleshoot some of the experimental stages (e.g. chromatin preparation and DNA elution conditions) which affect the signal-to-noise ratio. The degree of background binding may not be uniform across samples. Therefore, accurate determination of the background can have large effects on the final fold change. Moreover, the internal spike-in control strategy provides a simple means of normalising across samples and of reducing variability.

For several reasons, we believe that ChIP-ddPCR plus spike-in normalisation may be of particular value to the endocrine researcher. A highly sensitive technique, ddPCR has the potential to detect ChIP DNA from low input samples (e.g. clinical samples) with greater precision. This sensitivity is similarly valuable when working with low replicate numbers and low abundance proteins such as nuclear hormone receptors. ChIP has been considered a qualitative or semi-quantitative technique at best; absolute quantification with ddPCR followed by normalisation to an internal spike-in control permits comparison between treatments or between genotypes (Caratti et al. 2018). It also allows confident evaluation of the success of a ChIP reaction before samples are submitted for sequencing (we typically check a small, diluted aliquot of ChIP DNA for positive and negative signal prior to sequencing and only submit samples with a fivefold or greater enrichment of positive signal over negative). Sequencing is expensive and is typically done with small numbers of replicates (current guidelines suggest two biological replicates; ENCODE 2017); thus, it is particularly important to ensure that submitted samples are of good quality. ChIP-ddPCR does have the downsides of being currently more costly than ChIP-qPCR, and of being less amenable to large sample numbers, but these factors may improve as the technology progresses. Furthermore, it may save time and expense incurred in repeating experiments or in sequencing poor quality samples. By correcting for some of the variation introduced by technical factors, the spike-in strategy may also reduce the incidence of false-positive or false-negative results, thus improving reproducibility of ChIP experiments.

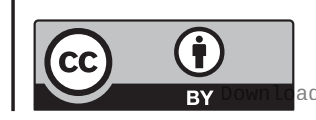




\section{Supplementary data}

This is linked to the online version of the paper at https://doi.org/10.1530/ JME-18-0243.

\section{Declaration of interest}

David Ray is a Senior Editor of Journal of Molecular Endocrinology. David Ray was not involved in the review or editorial process for this paper, on which he/she is listed as an author. The other authors have nothing to disclose.

\section{Funding}

This work was supported by MRC programme grant MR/P011853/1 and MRC Clinical Research Training Fellowship MR/N021479/1 awarded to A L H. D W R is a Wellcome Senior Investigator 107849/z/15/z.

\section{Author contribution statement}

A L H, N N, M B and T M P conducted experiments. A L H, D W R and T M P designed experiments. A L H and T M P analysed data and wrote the first draft of the paper. D W R supervised the project. All authors contributed to the final review of the paper.

\section{References}

Bonhoure N, Bounova G, Bernasconi D, Praz V, Lammers F, Canella D, Willis IM, Herr W, Hernandez N, Delorenzi M, et al. 2014 Quantifying ChIP-seq data: a spiking method providing an internal reference for sample-to-sample normalization. Genome Research $\mathbf{2 4}$ 1157-1168. (https://doi.org/10.1101/gr.168260.113)

Caratti G, Iqbal M, Hunter L, Kim D, Wang P, Vonslow RM, Begley N, Tetley AJ, Woodburn JL, Pariollaud M, et al. 2018 REVERBa couples the circadian clock to hepatic glucocorticoid action. Journal of Clinical Investigation 128 4454-4471. (https://doi.org/10.1172/ JCI96138)

Cebola I, Rodríguez-Seguí SA, Cho CH-H, Bessa J, Rovira M, Luengo M, Chhatriwala M, Berry A, Ponsa-Cobas J, Maestro MA, et al. 2015 TEAD and YAP regulate the enhancer network of human embryonic pancreatic progenitors. Nature Cell Biology 17 615-626. (https://doi. org/10.1038/ncb3160)

Chen F, Ding X, Feng Y, Seebeck T, Jiang Y \& Davis GD 2017 Targeted activation of diverse CRISPR-Cas systems for mammalian genome editing via proximal CRISPR targeting. Nature Communications $\mathbf{8}$ 14958. (https://doi.org/10.1038/ncomms14958)

Chen K, Hu Z, Xia Z, Zhao D, Li W \& Tyler JK 2015 The overlooked fact: fundamental need for spike-in control for virtually all genomewide analyses. Molecular and Cellular Biology 36 662-667. (https:// doi.org/10.1128/MCB.00970-14)

Du M \& Wang L 2016 3C-digital PCR for quantification of chromatin interactions. BMC Molecular Biology 17 23. (https://doi.org/10.1186/ s12867-016-0076-6)
Egan B, Yuan CC, Craske ML, Labhart P, Guler GD, Arnott D, Maile TM, Busby J, Henry C, Kelly TK, et al. 2016 An alternative approach to ChIP-seq normalization enables detection of genomewide changes in histone $\mathrm{H} 3$ lysine 27 trimethylation upon EZH2 inhibition. PLOS ONE 11 e0166438. (https://doi.org/10.1371/ journal.pone.0166438)

ENCODE project 2017. In ENCODE experimental guidelines for ENCODE3 ChIP-Seq. (available at: https://www.encodeproject.org/about/ experiment-guidelines/). Accessed on 18 January 2019.

Hindson BJ, Ness KD, Masquelier DA, Belgrader P, Heredia NJ, Makarewicz AJ, Bright IJ, Lucero MY, Hiddessen AL, Legler TC, et al. 2011 High-throughput droplet digital PCR system for absolute quantitation of DNA copy number. Analytical Chemistry 83 8604-8610. (https://doi.org/10.1021/ac202028g)

Jordán-Pla A \& Visa N 2018 Considerations on experimental design and data analysis of chromatin immunoprecipitation experiments. Methods in Molecular Biology 1689 9-28. (https://doi.org/10.1007/9781-4939-7380-4_2)

Kim W, Ludlow AT, Min J, Robin JD, Stadler G, Mender I, Lai TP, Zhang N, Wright WE \& Shay JW 2016 Regulation of the human telomerase gene tert by telomere position effect - over long distances (TPE-OLD): implications for aging and cancer. PLoS Biology 14 e2000016. (https://doi.org/10.1371/journal.pbio.2000016)

Link N, Kurtz P, O'Neal M, Garcia-Hughes G \& Abrams JM 2013 A p53 enhancer region regulates target genes through chromatin conformations in cis and in trans. Genes and Development $\mathbf{2 7}$ 2433-2438. (https://doi.org/10.1101/gad.225565.113)

Orlando DA, Chen MW, Brown VE, Solanki S, Choi YJ, Olson ER, Fritz CC, Bradner JE \& Guenther MG 2014 Quantitative ChIP-Seq normalization reveals global modulation of the epigenome. Cell Reports 9 1163-1170. (https://doi.org/10.1016/j.celrep.2014.10.018)

Phuc Le P, Friedman JR, Schug J, Brestelli JE, Parker JB, Bochkis IM \& Kaestner KH 2005 Glucocorticoid receptor-dependent gene regulatory networks. PLoS Genetics 1 e16. (https://doi.org/10.1371/ journal.pgen.0010016)

Ross-Innes CS, Stark R, Teschendorff AE, Holmes KA, Ali HR, Dunning MJ, Brown GD, Gojis O, Ellis IO, Green AR, et al. 2012 Differential oestrogen receptor binding is associated with clinical outcome in breast cancer. Nature 481 389-393. (https://doi. org/10.1038/nature10730)

Schubert C 2018 Technology feature | ChIP off the old block: Beyond chromatin immunoprecipitation. Science 362 1193.2-1193. (https:// doi.org/10.1126/science.362.6419.1193-b)

Taylor SC, Laperriere G \& Germain H 2017 Droplet digital PCR versus qPCR for gene expression analysis with low abundant targets: from variable nonsense to publication quality data. Scientific Reports $\mathbf{7}$ 2409. (https://doi.org/10.1038/s41598-017-02217-x)

Wong D, Lee W, Humburg P, Makino S, Lau E, Naranbhai V, Fairfax BP, Chan K, Plant K \& Knight JC 2014 Genomic mapping of the MHC transactivator CIITA using an integrated ChIP-seq and genetical genomics approach. Genome Biology 15 494. (https://doi.org/10.1186/ s13059-014-0494-z)

Zwart W, Koornstra R, Wesseling J, Rutgers E, Linn S \& Carroll JS 2013 A carrier-assisted ChIP-seq method for estrogen receptor-chromatin interactions from breast cancer core needle biopsy samples. $B M C$ Genomics 14 232. (https://doi.org/10.1186/1471-2164-14-232)

Received in final form 6 February 2019

Accepted 11 March 2019 https://jme.bioscientifica.com https://doi.org/10.1530/JME-18-0243 (c) 2019 The authors Published by Bioscientifica Ltd. Printed in Great Britain

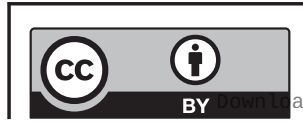

This work is licensed under a Creative Commons Attribution 4.0 International License. ded from Bioscientifica.com at 04/26/2023 05:51:12AM 